\title{
Optimal design of dynamic multi-axis force/torque sensor
}

\author{
A. Bicchi ${ }^{1,2}$ \\ A. Caiti ${ }^{1,3}$ \\ D. Prattichizzo ${ }^{1,3}$ \\ ${ }^{1}$ Centro "E. Piaggio", Università di Pisa. \\ ${ }^{2}$ Dip. Sistemi Elettrici e Automazione, Università di Pisa. \\ ${ }^{3}$ Dip. di Ingegneria dell'Informazione, Università di Siena. \\ Email: bicchi@ing.unipi.it, andy@dist.unige.it, prattichizzo@ing.unisi.it
}

\begin{abstract}
The measurement of time-varying forces and torques applied to flexible mechanical structures cannot be pursued by relying on quasi-static approximations. However the problem can be cast as a problem of dynamic system inversion, provided reliable models of the dynamic relationship among force/torques and measurement gauges are available. Within this framework, it is possible in particular to define optimality criteria for gauge placements in order to guide the design of such dynamic force/torque sensing devices. Experimental results show that the approach proposed is indeed capable of reconstructing forces, torques and point of contact applied to a flexible beam with a rigid part.
\end{abstract}

\section{Introduction}

A typical force and torque sensor consists of a deformable structure solicited by forces and torques applied on a specially shaped structure which is usually rigid, fig.1. The informative output is represented by deformations sensed by strain gauges glued on the deformable part. The estimation of the applied

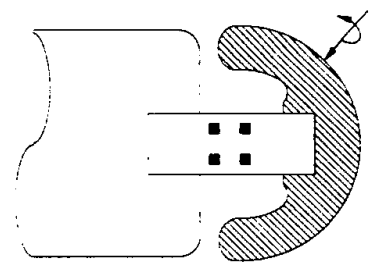

Figure 1: Force/torque sensors have a deformable part where strain gauges are glued and a rigid one where force and torques are applied.

force/torques from the strain measurements is customarily obtained by assuming a quasi-static relationship between the force applied and the strain. This assumption is maintained even if the force is time varying, provided the mechanical structure is rigid enough to make the effects of ribration in the structure itself negligible. Such is the case e.g. for most six-axis force/torque sen- sors mounted at the end-effector of robot manipulators.

Situations may arise, however, in which the flexibility of the mechanical structure is such that the measured strains include components due to the vibration of the structure, and consequently the estimation of the applied, time-varying, force cannot be done by using quasi-static relationships. To address this issue, the authors have considered dynamic force/torque sensing, i.e., measurement systems for recovering time varying forces and torques from the strain measurements by exploiting the dynamic nature of the force/strain relationship [1]. The approach is as follows. Model the natural modes of vibrations of the mechanical structure via elasticity theory, derive a finite dimensional approximation of the solution by truncating the modal expansion of the solution and describe this approximate system by means of a dynamic system, for instance in a state space setting. Finally address the problem of reconstructing the system input given the measured output, as a deconvolution problem or, more in general, as a problem of system inversion.

Once dynamic force/torque sensing is cast as a system inversion problem, several issues must be addressed. In particular, the position of the strain gauges on the mechanical structure does influence the structure of the input-output relationship and one may ask which are positions to be preferred for the strain gauges placement, in order to facilitate the inversion task. In this paper such problem is stated in an optimal design framework. Moreover, it would be desirable that the input reconstruction could be carried out introducing the shortest possible delay of the measurements with respect to the phenomena; furthermore, to guarantee some margin of stability for the system's inverse, a corresponding minimu-phase margin of the system to be inverted needs to be ensured.

Flexible mechanical systems with non co-located actuation and measurement points are known to exhibit non minimum phase properties. The optimal sensor design problem has then to compromise between three different factors:

- the accuracy of the approximated model chosen for the system;

- the maximization of a measure of inversion accuracy, 


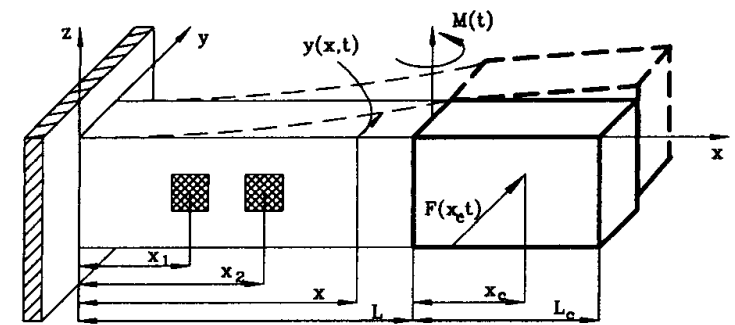

Figure 2: The physical system considered consists of a flexible beam of length $L$ and a rigid bar of length $L_{c}$. The beam is fixed at the left end $(x=0)$ while the right end $(x=L)$ is rigidly attached to the rigid bar.

and

- the minimum phase requirements.

In [1] authors considered the design of single-input dynamic force sensors. In this paper, a multi-input, multi-output model is proposed and discussed. The MIMO case addressed here allows in particular to independently determine the unknown intensities of a force and a moment applied to the mechanical structure, or, equivalently, to the intensity of the force and the position of the its application point.

The paper is organized as follows. In the next section the physical setting is described, and a state space model is derived. In Section 3 the design procedure is illustrated, in particular with respect to the optimality criterion. The specific inversion algorithm employed for in-line input reconstruction is described in Section 4. Experimental results are reported in Section 5, showing an excellent agreement between measured data, reconstructed input and theoretical expectations. Conclusions are finally given in Section 6.

\section{State space model of the flexible sensor}

In this paper we consider measurements of the deformations of a mechanical system comprised of a slender beam, clamped at one end, and with a rigid part fixed at the other extremity. This particular structure lends itself to rather simple analytical treatment, and is also easy to build to obtain experimental validation of results. On the other hand, it can also be considered to be a paradigm of more general multi-axis force/torque sensors, being such devices typically characterized by a deformable part (where measurements are taken) clamped on one side and supporting a rigid part on theother end. Forces and torques to be measured are exerted on the rigid part, while the induced deformations on the deformable structure are sensed by a certain number of strain-gauges, fig.1. The measurement strain signals are used to retrieve information on the input forces according to an inversion algorithm of the system. Usually inversion algorithms are based on quasi-static input/output relationships and do not allow the estimation of time-varying forces and torques. In cases where there exists significant elasticity in the sensor structure, or to the purpose of increasing the effective bandwidth of signals reconstructed by the sensor, the exploitation of information contained in the transients of measured strain-gauges signals is instrumental [1]. To pursue this objective, an input/output dynamic model of the force/torque sensors is needed.

The 2-input, 2-measurement dynamic sensor studied in this paper, described in fig.2, is comprised of a flexible beam of length $L$ and a rigid bar of length $L_{c}$. Both the beam and the bar are assumed isotropic, and of total mass $m$ and $m_{c}$, respectively. The beam is clamped at the left end $(x=0)$ while the right end $(x=L)$ is rigidly attached to the bar. The rigid bar is subject to a time-varying force $F(t)$ exerted at a varying distance $x_{c}$ from the end of the flexible beam, cf. fig.2. The beam is excited by an equivalent force $F(t)$ and moment $M(t)=$ $F(t) x_{c}(t)$ at its extremity $x=L$, and deformations are measured by two strain gauges, placed at positions $x_{1}$ and $x_{2}$ along the beam. These measurements are used to estimate $F(t)$ and $M(t)$ and, indirectly, the application point $x_{c}$.

To simplify the analysis, let us assume that the force $F$ applied at $x_{c}$ lies on the neutral axis of the beam, so that only transverse vibrations $y(x, t)$ are solicited, fig. 2 .

The transverse vibration dynamics of a slender monodimensional beam is a well studied system [2] governed by the Euler-Bernoulli equations:

$$
E I \frac{\partial^{4} y}{\partial x^{4}}+2 \xi \frac{\partial^{4}}{\partial x^{4}} \frac{\partial}{\partial t} y+m \frac{\partial^{2} y}{\partial t^{2}}=0
$$

where $E I, \xi$ and $m$ are the stiffness, structural damping and mass of the beam.

The first two boundary conditions at $x=0$ are simply

$$
y(x, t)=0, \quad \frac{\delta y(x, t)}{\delta x}=0 .
$$

To derive the other two boundary conditions, the interaction between beam and bar at $x=L$ must be taken into account.

Because of the rigidity of the bar, force $F(t)$ can be considered as applied in $x=L$ and the force balance equation at $x=L$ is

$$
m_{c} \ddot{y}_{G}(t)+E I \frac{\partial^{3} y(x, t)}{\partial x^{3}}=F(t)
$$

where $\ddot{y}_{G}$ is the center-of-mass acceleration of the bar. The equilibrium of moments about the $z$-axis through $x=L$ is obtained as

$$
I_{G} \dot{\omega}(t)-r_{O G} m_{c} \ddot{y}_{G}(t)+E I \frac{\partial^{2} y(x, t)}{\partial x^{2}}=M(t)
$$


where $I_{G}$ is the barycentral inertia of the rigid bar, $r_{O G}$ is the distance along the $x$-axis between the end of the beam and the center of gravity of the bar and $M(t)=F(t) x_{c}$.

It is well known ([2]) that the solution of the EulerBernoulli equation (1) with boundary condition as in (2), (4) and (3) can be expressed in terms of the normal modes decomposition as:

$$
y(x, t)=\sum_{k=1}^{\infty} q_{k}(t) Y_{k}(x)
$$

where the terms $q_{k}(t)$ play the role of a weight in time and the normal modes $Y_{k}(x), x \in[0, L]$ are defined as

$$
\begin{gathered}
Y_{k}(x)=\left(\sin \left(\alpha_{k} L\right)-\sinh \left(\alpha_{k} L\right)\right)\left(\sin \left(\alpha_{k} x\right)-\sinh \left(\alpha_{k} x\right)\right) \\
+\left(\cos \left(\alpha_{k} L\right)+\cosh \left(\alpha_{k} L\right)\right)\left(\cos \left(\alpha_{k} x\right)-\cosh \left(\alpha_{k} x\right)\right)
\end{gathered}
$$

being $\alpha_{k}(k=1, \cdots, \infty)$ the $k$-th solution of a nonlinear algebraic equation.

By truncating modal expansion (5) to the $N$-th mode, and by defining time weights and their derivative as the state vector:

$$
\mathbf{x}=\left[q_{1}(t), \cdots, q_{N}(t), \dot{q}_{1}(t), \cdots, \dot{q}_{N}(t)\right]^{T},
$$

the following system of ordinary differential equations is obtained after some algebraic computation

$$
\left\{\begin{array}{l}
\dot{\mathbf{x}}=A \mathbf{x}+B \mathbf{u} \\
\mathbf{y}=C \mathbf{x}
\end{array}\right.
$$

where $\mathbf{u}(t)=[F(t), M(t)]^{T}$ is the input vector of force and moment to be estimated, $\mathbf{y}=\left[s_{1}(t), s_{2}(t)\right]^{T}$ is the vector of measurement signals from the strain gauges placed at $x_{1}$ and $x_{2}$, and matrices $A \in \mathbb{R}^{2 N \times 2 N}, B \in$ $\mathbb{R}^{2 N \times 2}$ and $C \in \mathbb{R}^{2 \times 2 N}$ assume the following structures

$$
\begin{gathered}
A=\left[\begin{array}{cc}
0 & I_{N} \\
-\left(\frac{E I}{m}\right) \Lambda^{4} & -2 \frac{\xi}{m} \Lambda^{4}
\end{array}\right] \\
B=\left[0_{(2 \times N)} \mid\left[\begin{array}{lll}
Y_{1}(L) & \cdots & Y_{N}(L) \\
\frac{\partial^{2} Y_{1}(h)}{\partial x^{2}} & \cdots & \frac{\partial^{2} Y_{N}(h)}{\partial x^{2}}
\end{array}\right]\right]^{T} \\
C=\left[\left[\begin{array}{lll}
\frac{\partial^{2} Y_{1}\left(x_{1}\right)}{\partial x^{2}} & \ldots & \frac{\partial^{2} Y_{N}\left(x_{1}\right)}{\partial x^{2}} \\
\frac{\partial^{2} Y_{1}\left(x_{2}\right)}{\partial x^{2}} & \cdots & \frac{\partial^{2} Y_{N}\left(x_{2}\right)}{\partial x^{2}}
\end{array}\right] \mid 0_{(2 \times N)}\right]
\end{gathered}
$$

being matrix $\Lambda$ a $N \times N$ diagonal matrix whose $k$-th diagonal term is $\alpha_{k}$.

\section{Optimal Sensor Design}

The problem of estimating time varying force/torque input signals $\mathbf{u}(t)$ from the strain gauges measurements $\boldsymbol{y}(t)$, can be stated as a problem of input/output inversion for linear time invariant dynamic systems $[3,4]$.
Necessary and sufficient conditions for system (7) to be invertible have been described in a state space framework by $[4,3]$ in terms of a matrix $M \in \mathbb{R}^{4 N-1 \times 4 N}$, defined as

$$
M=\left[\begin{array}{cccc}
C B & 0 & \cdots & 0 \\
C A B & C B & \ddots & \vdots \\
\vdots & \ddots & \ddots & 0 \\
C A^{2 N-1} B & \cdots & \cdots & C B \\
C A^{2 N} B & \cdots & \cdots & C A B \\
\vdots & \ddots & \ddots & \vdots \\
C A^{4 N-1} B & \cdots & \cdots & C A^{2 N-1} B
\end{array}\right] .
$$

In particular, [4] proves that system (7) is invertible if and only if matrix $M$ is full column rank. Consider inputs $\mathbf{u}(t)$ that are analytic and have a Laplace transform admitting the Laurent expansion

$$
\mathcal{L}(\mathbf{u}(t))=\frac{1}{s}\left(\mathbf{u}_{o}+\frac{1}{s} \mathbf{u}_{1}+\frac{1}{s^{2}} \mathbf{u}_{2}+\cdots \frac{1}{s^{p}} \mathbf{u}_{p}+\cdots\right)
$$

where $\mathbf{u}_{p}=\left.\frac{d^{p} \mathbf{u}(t)}{d t^{p}}\right|_{t=0}$, and similarly for $\mathbf{y}(t)$ Define the class $\mathcal{U}$ of polynomial input signals as

$$
\mathcal{U}=\left\{\mathbf{u}(t) \mid \mathcal{L}(\mathbf{u}(t))=\frac{1}{s}\left(\mathbf{u}_{o}+\frac{1}{s} \mathbf{u}_{1}+\cdots \frac{1}{s^{2 N-1}} \mathbf{u}_{2 N-1}\right)\right\}
$$

For $u(t) \in \mathcal{U}$, the matrix $M_{p} \in \mathbb{R}^{2 p \times 4 N}$,

$$
M_{p}=\left[\begin{array}{cccc}
\multicolumn{4}{c}{M} \\
\hline C A^{4 N} B & \cdots & \cdots & C A^{2 N} B \\
C A^{4 N+1} B & \cdots & \cdots & C A^{2 N+1} B \\
\vdots & & & \vdots \\
C A^{p} B & \cdots & \cdots & C A^{p-2 N} B
\end{array}\right]
$$

with $p \geq 4 N-1$, maps the $2 N$ coefficients $\left(\mathbf{u}_{0}, \ldots, \mathbf{u}_{2 N-1}\right)$ of signals $\mathbf{u}(t) \in \mathcal{U}$ into the first $p$ coefficients $\left(\mathbf{y}_{1}, \ldots, \mathbf{y}_{p}\right)$ of the Laurent expansion of the Laplace transform of the corresponding $\mathbf{y}(t)$ output.

The relevance of the class of input signals $\mathcal{U}$ to the invertibility of dynamic systems is discussed in [3], where authors proved that system (7) is invertible if and only if $M_{p}(p \geq 4 N-1)$ is full column rank and that (from the Caley-Hamilton theorem) $\operatorname{rank}\left(M_{p}\right)=\operatorname{rank}(M)$. From (13), being $M=\left.M_{p}\right|_{p=4 N-1}$, it ensues that $M$ in (11) represents the invertible matrix with the minimum number of rows, i.e., the minimum number of derivatives (at $t=0$ ) of $y(t)$ that allow to retrieve $u(t) \in \mathcal{U}$. In discrete-time implementations of inversion algorithms, this means obtaining inversions with minimum delay, and therefore we will focus on maximizing the invertibility of $M$ through the sensor design.

In this paper, an optimal criterion for the design of dynamic force sensors consisting in maximizing the minimum singular values of $M$ that depends, through the output matrix $C$, upon the gauge positions $x_{1}$ and $x_{2}$, the free parameters of the dynamic sensor design, is 
considered. Maximizing the minimum singular value of the measurement matrix increases the accuracy of the inversion independently from the numerical algorithm that will be used, [8]. Note that the minimum singular value of $M$ depends upon parameter $N$, the number of modes, that must be fixed before applying the optimization procedure for the strain gauges placement.

The inversion accuracy is not the unique requirement of the sensor design. In fact, as regards the inversion algorithm, a stability requirement on the inversion algorithm must be added. In terms of transfer functions this is equivalent to require that, for the strain gauges positions $x_{1}$ and $x_{2}$ the transfer function $G(s)=C(s I-A)^{-1} B$ of system (7) must not have any zero with positive real part. The complete design criterion hence takes the form:

$$
\begin{aligned}
& \max _{x_{1}, x_{2} \in[0, L]} \sigma_{\min }\left(M\left(x_{1}, x_{2}\right)\right) \\
& \text { subject to: } \operatorname{Re}\left(z_{i}\right)<0 \quad \forall z_{i} \in z(G(s))
\end{aligned}
$$

where $\sigma_{\min }$ is the minimum singular value and $z(G(s))$ is the set of all transmission zeros of the transfer function $G(s)$.

The constrained optimization problem is usually solved by means of nonlinear programming methods. However in this simplified case with two free parameters, the problem can be tackled by exhaustive search and visual inspection.

It should be remarked that the minimum singular value and positions of the transfer function zeros change with the number $N$ of modes chosen to approximate the system dynamics [1]. This means that the optimal design of the sensor needs the a priori specification of the maximum number of modes that one wishes to invert for. Such a number may be determined by the dynamic range of the strain gauge, by the precision one wish to achieve and so forth. Notice that, as the number of modes increases, the admissible region (w.r.t. the minimum-phase requirement) shrinks progressively towards the free end point of the beam. Once the optimal sensor design has been chosen for a given modal approximation, using the sensor with a finer approximation (higher number of modes) would likely lead to violation of minimum-phase conditions, and instability of the sensor. An interesting problem of trading off modeling accuracy (increased by increasing the number of modes) for invertibility accuracy (decreased because the minimum-phase constraint grows tighter) ensues, which needs further investigation.

\section{Inversion Algorithm}

Several algorithms could be selected for system inversion. The constraint here is that the inversion is required in almost-real-time, i.e., with the minimum possible delay with respect to the measurement. The reg- ularized backward Euler algorithm proposed in [9] has been selected since it has a delay of one sampling step with respect to the measurement, and a built-in lowpass capability through the regularization parameters $\alpha$ that guarantees a certain degree of robustness with respect to the measurement noise.

From dynamic system ( 7 ), the following implicit system is built:

$$
\left[\begin{array}{ll}
I & 0 \\
0 & 0
\end{array}\right]\left[\begin{array}{c}
\dot{\mathbf{x}} \\
\dot{u}
\end{array}\right]=\left[\begin{array}{cc}
A & B \\
C & 0
\end{array}\right]\left[\begin{array}{l}
\mathbf{x} \\
u
\end{array}\right]+\left[\begin{array}{c}
0 \\
-I
\end{array}\right] y .
$$

The implicit system (15) can be written in the compact form $E \dot{w}=F \mathbf{w}+G y$, with obvious meanings. Notice that system (15) is obtained just rewriting the equations of system (7) as an implicit system. By exploiting the non-directionality of implicit representations, it is possible to exchange the role of input and output. The system (15) is solvable, i.e., admits unique solution, if and only if the system (7) is invertible [10].

According to [9], the discretized version of system (15) is obtained as

$$
\begin{gathered}
\left(E-\tau\left(\frac{1}{2}+\alpha\right) F\right) \mathbf{w}(j+1)=\left(E+\tau\left(\frac{1}{2}-\alpha\right) F\right) \mathbf{w}(j)+ \\
+\tau G\left[\left(\frac{1}{2}+\alpha\right)(y(j+1)-y(j))+y(j)\right]
\end{gathered}
$$

where $\tau$ is the sampling period, and $\alpha \geq 1 / 2$ is referred to as the regularization parameter. For $\alpha=1 / 2$ the method above turns into the Backward Euler method. As $\alpha$ increases, the solutions of the discretized equation (16) are low-pass filtered versions of the exact solution, with cut-off frequency progressively decreasing.

It has to be remarked that, in some cases, a stronger low-pass filtering is needed than that allowed by the regularized backward Euler. In these cases, the delay in response amounted to one step plus as many steps as the filter order.

\section{Experimental Results}

The experimental device is comprised of a steel beam of $10 \mathrm{~cm}$ length, $3 \mathrm{~cm}$ height and $0.2 \mathrm{~cm}$ depth, rigidly clamped at one end. The other end of the beam is fixed to an aluminum bar whose dimension are $10 \mathrm{~cm}$ (length), $3 \mathrm{~cm}$ (height) and $4 \mathrm{~cm}$ (depth).

A 3-modal approximation is used to derive the state space model of the dynamic sensor and the row index $p$ of $M_{p}$ is set to 12 . The optimization problem in two variables $\left(x_{1}\right.$ and $\left.x_{2}\right)$ is reduced to a one-parameter problem by fixing the first strain gauge as close as possible to the clamped end (actually, $x_{1}=2 \mathrm{~cm}$ because of hardware constraints). To choose the position of the second strain gauge, the behaviour of the minimum singular value of $M_{p}$ as a function of the position $x_{2}$ of the second strain gauge is computed and plotted in fig.3-a (similar plots, with lower index values, are obtained by choosing larger values of $x_{1}$ ). Constraints on the stability of the inversion system are reported in fig.3-b. The 


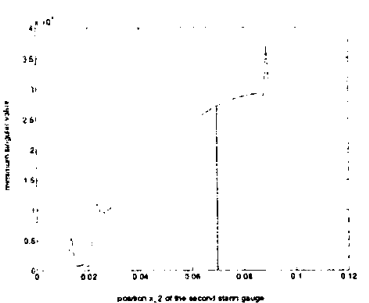

a)

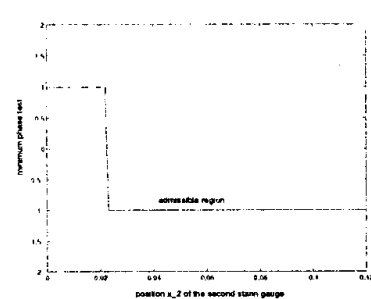

b)
Figure 3: Cost function, i.e. minimum singular value of $M_{12}$ (a), and constraints on zeros (b) for the optimal design problem, computed with a 3modes model when the first strain gauge is fixed at $x_{1}=2 \mathrm{~cm}$.

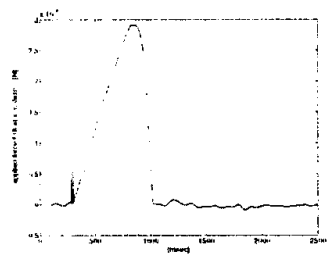

a)

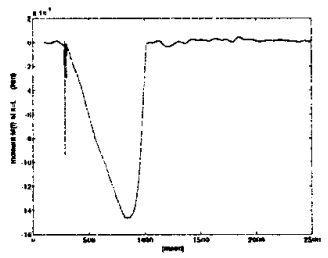

b)
Figure 4: Time-varying force (a) applied at $x_{c}=5 \mathrm{~cm}$ and (b) related moment $M(t)=F(t) x_{c}$.

sign of the maximum real part of the zeros of $G(s)$ is reported. The sign function takes on value -1 if the zeros of $G(s)$ have all negative real part (admissible region) and +1 region if at least one zero of $G(s)$ has a positive real part (inadmissible region). It appears that if $x_{2}>2.3 \mathrm{~cm}$ transfer function $C(s I-A)^{-1} B$ does not exhibit zeros on the right half plane and the inversion algorithm is stable.

Because of hardware constraints on the flexible beam of the experimental device, we choose $x_{2}=7 \mathrm{~cm}$ as the position of the second strain gauge, cf. fig.3-a. Although this is a slightly sub-optimal solution of the optimization problem addressed in (14), the experimental results are satisfactory as it will be shown later on.

Two different experiments have been made on the force/torque dynamic sensor.

In the first experience, the rigid bar is solicited at $x_{c}=$ $5 \mathrm{~cm}$ with the force $F(t)$ and moment $M(t)$ reported in fig.4. The force $F(t)$ has been applied and measured by means of a special precision measurements instrument consisting of a hammer and a piezoelectric sensor. The moment $M(t)$ at $x=L$ is given by the product of the force $F(t)$ and the moment arm $x_{c}=5 \mathrm{~cm}$.

The signals of the two strain gauges placed at $x_{1}=2 \mathrm{~cm}$ and $x_{2}=7 \mathrm{~cm}$ are reported in fig. 5 .

Direct measurements and a least mean square procedure has been used to identify the physical parameters

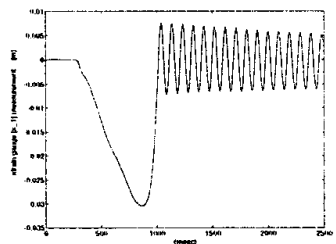

a)

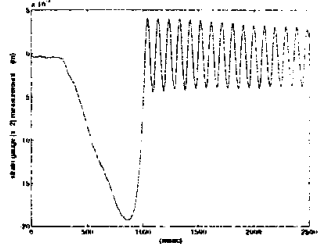

b)
Figure 5: Measurement signals of strain gauge glued at $x_{1}=2 \mathrm{~cm}(\mathrm{a})$ and of strain gauge glued at $x_{2}=$ $7 \mathrm{~cm} \mathrm{(b).}$

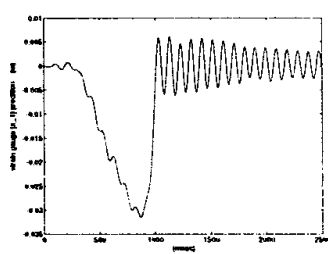

a)

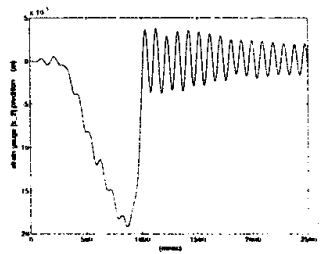

b)
Figure 6: Prediction of strain gauge output at $x_{1}=2 \mathrm{~cm}$ (a) and of strain gauge at $x_{2}=7 \mathrm{~cm}(\mathrm{~b})$.

of the steel beam and aluminum bar. Both the hammer input signal and the strain gauge output signals have been acquired with a 12-bit A/D converter having a sampling rate of $2 \mathrm{KHz}$. Fig. 6 describes the prediction of the strain gauge outputs obtained with the 3-modes approximation and the input signal of fig.4-a. A simple inspection of fig. 6 and fig. 5 shows that physical parameters of the system have been identified with satisfactory approximation, and also that three modes are enough to describe the system behaviour.

The estimation of force and moment obtained by the inversion algorithm described in Section 4 is shown in fig.7-a and fig.7-b, respectively.

The estimated inputs must be directly compared with the real inputs reported in fig.4. The estimates of input force $F(t)$ and moment $M(t)$ are in excellent agreement with the measured inputs signal, showing good noise rejection properties and sufficient insensitivity to model

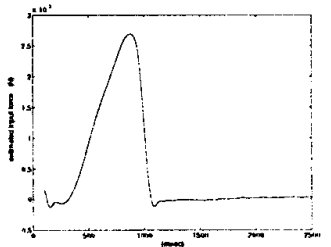

a)

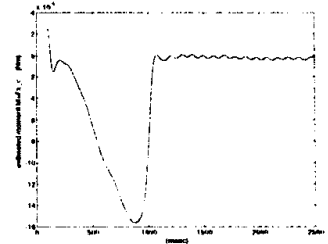

b)
Figure 7: Estimation of input force (a), moment (b). 


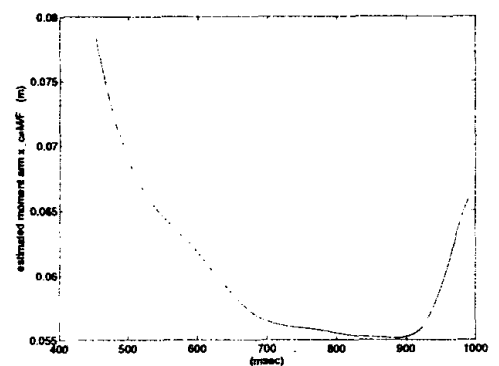

Figure 8: Estimation of contact point whose actual value is $x_{c}=5 \mathrm{~cm}$.

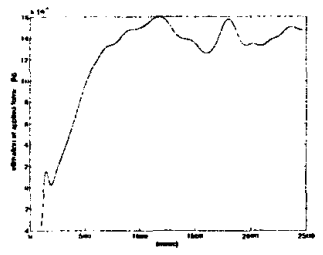

a)

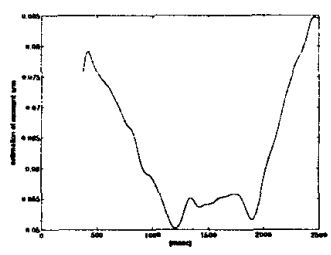

b)
Figure 9: Estimation of contact force (a) and of contact point (b) when a force is applied by hand with a time-varying application point. No measurement of applied force is available.

mismatch. From the relationship $M(t)=F(t) x_{c}$, it is an easy matter to retrieve information on the contact point, fig. 8 , whose actual value is $x_{2}=5 \mathrm{~cm}$. Observe that due to the impulsive nature of the input signals the transient information is paramount and quasi-static relationships are not useful at all.

In the second experiment a force $F(t)$ is applied by hand at a time-varying application point which moves from $x_{c}=5 \mathrm{~cm}$ to $x_{c}=9 \mathrm{~cm}$ and return. The estimated force and the estimation of the contact point $x_{c}(t)$ are reported in fig.9-a and in fig.9-b, respectively.

\section{Conclusions}

In this paper the optimal design of dynamic multi-axis force/torque sensors was pursued. In order to estimate time varying forces and torques, transient information have been exploited by means of a dynamic model of the sensor device. By optimality is meant the optimal placement of strain gauges for the estimation of force and torque inputs. The case of a monodimensional flexible beam with a rigid bar was considered and experimental results were presented. In the experiments both the input force and moment or, equivalently, the input force and the application point are retrieved by informative outputs. Experimental results show that it is possible to obtain robust solutions to the inversion problem, notwithstanding the use of an approximated model in building of the inverse system. Future developments of this work are foreseen on further investigation on the estimation algorithm and on the optimality criterion.

\section{References}

[1] A. Bicchi, A. Caiti, and D. Prattichizzo, "Dynamic force/torque sensors: Theory and experiment," in Proc. IEEE 8th International Conference on Advanced Robotics, September 1997.

[2] L. Meirovitch, Analytical methods in vibration, Macmillan Publishing Co., Inc., New York, 1967.

[3] M.K. Sain and J.L. Massey, "Invertibility of linear time invariant dynamical systems," IEEE Trans. Autom. Contr., vol. 14, pp. 141-149, 1969.

[4] R.W. Brockett and M. Mesarovich, "The reproducibility of multivariable systems," Jour. Math. Anal. Appl., vol. 11, pp. 548-563, 1965.

[5] L.M. Silverman, "Inversion of multivariable linear systems," IEEE Trans. Autom. Contr., vol. 14, pp. 270-276, 1969.

[6] P.J. Moylan, "Stable inversion of linear systems," IEEE Trans. Autom. Contr., vol. 22, pp. 74-78, 1977.

[7] S. Tan and J. Vandewalle, "Inversion of singular systems," IEEE Trans. Circuit. Sys., vol. 35, pp. 583$587,1988$.

[8] A. Bicchi and G. Canepa, "Optimal design of multivariate sensors," Meas. Sci. Technol., vol. 5, pp. 319-332, 1994.

[9] A. Caiti and G. Cannata, "Stabilization of spectral methods for the analysis of singular systems using piecewise constant basis functions," Circ. Sys. Sig. Proc., vol. 14, pp. 299-316, 1995.

[10] F.L. Lewis, M.A. Christodoulou, and B. Mertzios, "System inversion using orthogonal functions," Circ. Sys. Sig. Proc., vol. 8, pp. 347-362, 1987. 